\title{
On equivalence of exponential and asymptotic stability under changes of variables
}

\author{
Lars Grüne \\ Fachbereich Mathematik \\ J.W. Goethe-Universität \\ Postfach 111932 \\ 60054 Frankfurt a.M., Germany \\ gruene@math.uni-frankfurt.de \\ Fabian Wirth \\ Zentrum für Technomathematik \\ Universität Bremen \\ 28334 Bremen, Germany \\ fabian@math.uni-bremen.de
}

\author{
Eduardo D. Sontag \\ Department of Mathematics \\ Rutgers University \\ New Brunswick, NJ 08903, USA, \\ sontag@control.rutgers.edu
}

\begin{abstract}
We show that uniformly global asymptotic stability for a family of ordinary differential equations is equivalent to uniformly global exponential stability under a suitable nonlinear change of variables.
\end{abstract}

\section{Introduction}

Lyapunov's notion of (global) asymptotic stability of an equilibrium is a key concept in the qualitative theory of differential equations and nonlinear control. In general, a far stronger property is that of exponential stability, which requires decay estimates of the type " $\|x(t)\| \leq$ $c e^{-\lambda t}\|x(0)\| . "$ In this paper, we show that, for differential equations evolving in finite-dimensional Euclidean spaces $\mathbb{R}^{n}$ (at least in spaces of dimensions $\neq 4,5$ ) the two notions are one and the same under coordinate changes.

Of course, one must define "coordinate change" with care, since under diffeomorphisms the character of the linearization at the equilibrium is invariant. However, if we relax the requirement that the change of variables be smooth at the origin, then all obstructions disappear. The basic ingredient of the construction we are about to present relies on the existence of smooth Lyapunov functions $V$. The coordinate transformations are constructed via "projecting" along the gradient flow of $V$ onto a level set $V^{-1}(c)$. The result now relies on the fact that this level set is diffeomorphic to the standard sphere, which is true except for those cases where the Poincaré conjecture is still open. This explains why we have to exclude $n=4,5$ from our statements.

Closely related to our work is the fact that all asymptotically stable linear systems are equivalent (in the sense just discussed) to $\dot{x}=-x$; see e.g. [1].

\section{Problem Statement}

Throughout the paper, $\|\cdot\|$ denotes the usual Euclidean norm, and "smooth" means $C^{\infty}$. For a differentiable function $V: \mathbb{R}^{n} \rightarrow \mathbb{R}$ the expression $L_{f_{d}} V(x)$ denotes the directional derivative 
$D V(x) f(x, d)$. We consider the family of differential equations

$$
\dot{x}(t)=f(x(t), d(t))
$$

where $f: \mathbb{R}^{n} \times D \rightarrow \mathbb{R}^{n}$ is continuous and for $x \neq 0$ locally Lipschitz continuous in $x$, where the local Lipschitz constants can be chosen uniformly in $d \in D \subseteq \mathbb{R}^{m}$. We assume that $D$ is compact and that $f(0, d)=0$ for all $d \in D$ and let $\mathcal{D}$ denote the set of measurable functions from $\mathbb{R}$ to $D$. Then we say that the zero state is uniformly globally asymptotically stable (UGAS) if there exists a class $\mathcal{K} \mathcal{L}$ function ${ }^{1} \beta$ such that, for each $d(\cdot) \in \mathcal{D}$, every maximal solution is defined for all $t \geq 0$ and

$$
\|\phi(t, x, d(\cdot))\| \leq \beta(\|x\|, t), \quad \forall t \geq 0 .
$$

Note that while our general assumptions on $f$ do not guarantee uniqueness of solutions through zero, assumption $(2.2)$ implies that $\phi(t, 0, d) \equiv 0$ is the unique solution with initial condition $x=0$, for all $d \in \mathcal{D}$ and thus the same is true for every initial condition.

An apparently stronger formulation of (2.2) is the following. We call the zero position of (2.1) uniformly globally exponentially stable (UGES), if there exist constants $c \geq 1, \lambda>0$ such that for all $d(\cdot) \in \mathcal{D}$

$$
\|\phi(t, x, d(\cdot))\| \leq c e^{-\lambda t}\|x\|, \quad \forall t \geq 0 .
$$

If the origin is no common fixed point for all values $d \in D$ then (2.2) is impossible. In this case, however, still a useful notion of stability is possible which is known as input-to-state stability. For this stability concept similar results to those discussed in this paper can be obtained, [3]. Extending the concepts in $[1$, p. 207] to our nonlinear setting, we will call a homeomorphism $T: \mathbb{R}^{n} \rightarrow \mathbb{R}^{n}$ a change of variables if $T(0)=0, T$ is $C^{1}$ on $\mathbb{R}^{n}$, and $T$ is diffeomorphism on $\mathbb{R}^{n} \backslash\{0\}$ (i.e., the restrictions of $T$ and of $T^{-1}$ to $\mathbb{R}^{n} \backslash\{0\}$ are both smooth). Given a change of variables $T$ and a system (2.1), we may consider the transformed system

$$
\dot{y}(t)=\tilde{f}(y(t), d(t)),
$$

where, by definition, $\tilde{f}(y, d)=D T\left(T^{-1}(y)\right) f\left(T^{-1}(y), d\right)$. In other words, system (2.4) is obtained from the original system by means of the change of variables $y=T(x)$. Observe that the new system again satisfies the general requirements.

It is our aim to show that for dimensions $n \neq 4,5$ the following assertions are true. Given a system of the form (2.1) satisfying (2.2) there exists a transformed system that satisfies (2.3). In this sense, global asymptotic stability is equivalent to global exponential stability under nonlinear changes of coordinates. Furthermore, one may obtain transformed systems where the constants defining the exponential stability property can be chosen to be the special values $c=\lambda=1$.

\section{Statement of Results}

The main tool for our construction of $T$ is the use of an appropriate Lyapunov function $V$. In fact, we can obtain $T$ for a whole class of functions as stated in the following proposition. Recall that a function $V: \mathbb{R}^{n} \rightarrow \mathbb{R}$ is called positive definite if $V(0)=0$ and $V(x)>0$ for all $x \neq 0$, and proper if the set $\{x \mid V(x) \leq \beta\}$ is bounded for each $\beta \geq 0$.

\footnotetext{
${ }^{1}$ As usual, we call a function $\alpha:[0, \infty) \rightarrow[0, \infty)$ of class $\mathcal{K}$, if it satisfies $\alpha(0)=0$ and is continuous and strictly increasing (and class $\mathcal{K}_{\infty}$ if it is unbounded), and we call a continuous function $\beta:[0, \infty)^{2} \rightarrow[0, \infty)$ of class $\mathcal{K} \mathcal{L}$, if it is decreasing to zero in the second and of class $\mathcal{K}$ in the first argument.
} 
Proposition 3.1 Let $n \neq 4,5$ and let $V: \mathbb{R}^{n} \rightarrow \mathbb{R}$ be a proper, positive definite $C^{1}$ function. Assume furthermore that $V$ is smooth on $\mathbb{R}^{n} \backslash\{0\}$ with nonvanishing gradient. Then there exists a class $\mathcal{K}_{\infty}$ function $\gamma$ which is smooth on $(0, \infty)$ and satisfies $\gamma(s) / \gamma^{\prime}(s) \geq s$ and a change of variables $T: \mathbb{R}^{n} \rightarrow \mathbb{R}^{n}$ with $T(0)=0$ such that

$$
\tilde{V}(y):=V\left(T^{-1}(y)\right)=\gamma(\|y\|)
$$

Outline of proof: Let $\psi$ denote the smooth flow determined by

$$
\dot{x}=\frac{\nabla V(x)^{\prime}}{\|\nabla V(x)\|^{2}}
$$

Fix $c>0$ and define the smooth map $\pi: \mathbb{R}^{n} \backslash\{0\} \rightarrow V^{-1}(c)$ by $\pi(x)=\psi(c-V(x), x)$. Now observe that the properties of $V$ imply that $V^{-1}(c)$ is a homotopy sphere (cf. also [6, Discussion after Theorem 1.1]), so that $V^{-1}(c)$ is diffeomorphic to $\mathbb{S}^{n-1}$ for $n \neq 4,5$ ( see [2] for $n=1,2,3$, [5, S9, Proposition A] for $n \geq 6)$. Now $T$ is given by

$$
T(0)=0, \text { and } T(x)=\gamma^{-1}(V(x)) S(\pi(x)), \quad x \neq 0 .
$$

It is straightforward to see that $T$ satisfies (3.5). For the remaining statements see [3].

Theorem 3.2 Let $n \neq 4,5$ and consider any system (2.1) on $\mathbb{R}^{n}$ which is UGAS (2.2). Then, (2.1) can be transformed into a system (2.4) that is UGES (2.3). In particular, the constants in (2.3) can be chosen to be $c=1, \lambda=1$.

Outline of proof: Under our assumptions, by [4, Theorem 2.9, Remark 4.1] there exists a smooth function $V: \mathbb{R}^{n} \rightarrow \mathbb{R}$ for (2.1) such that

$$
L_{f_{d}} V(x) \leq-\alpha_{1}(\|x\|) \text { and } \alpha_{2}(\|x\|) \leq V(x) \leq \alpha_{3}(\|x\|) .
$$

for some class $\mathcal{K}_{\infty}$ functions $\alpha_{1}, \alpha_{2}, \alpha_{3}$. ¿From this it is possible to construct a $C^{1}$ class $\mathcal{K}_{\infty}$ function $\rho$ on $[0, \infty)$ which is smooth on $(0, \infty)$ with $\rho^{\prime}(0)=0$ and for which $W(x):=\rho(V(x))$ satisfes

$$
L_{f_{d}} W(x) \leq-W(x)
$$

Applying Proposition 3.1 to $W$, we obtain for each $d \in D$ and $y \neq 0$

$$
\langle\tilde{f}(y, d), y\rangle=\frac{\|y\|}{\gamma^{\prime}(\|y\|)} L_{\tilde{f}_{d}} \tilde{W}(y) \leq-\frac{\|y\|}{\gamma^{\prime}(\|y\|)} \tilde{W}(y)=-\frac{\|y\|}{\gamma^{\prime}(\|y\|)} \gamma(\|y\|) \leq-\|y\|^{2} .
$$

This immediately yields the desired exponential estimate.

\section{References}

[1] V.I. Arnol'd. Ordinary Differential Equations, Springer-Verlag, Berlin, 1992.

[2] B.A. Dubrovin, A.T. Fomenko, and S.P. Novikov. Modern Geometry - Methods and Applications, Part III: Introduction to Homology Theory, Springer-Verlag, New York, 1990.

[3] L. Grüne, E.D. Sontag and F.R. Wirth. Asymptotic stability equals exponential stability, and ISS equals finite energy gain-if you twist your eyes. Syst. Contr. Lett. (1999) to appear.

[4] Y. Lin, E.D. Sontag, and Y. Wang. A smooth converse Lyapunov theorem for robust stability. SIAM J. Control Optim. 34(1996): 124-160.

[5] J. Milnor. Lectures on the h-Cobordism Theorem, Princeton Mathematical Notes, Princeton Univ. Press, Princeton, NJ. 1965.

[6] F.W. Wilson. The structure of the level surfaces of a Lyapunov function. J. Differ. Equations 3 (1967): 323-329. 